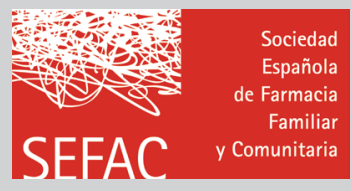

\title{
Creando puentes para un mejor abordaje de la cronicidad
}

Lola Murillo', José Miguel Ruiz²

1. Vicepresidenta de SEFAC. 2. Coordinador del documento Modelo de coordinación médico-farmacéutico para la atención al paciente con enfermedad crónica en atención primaria.

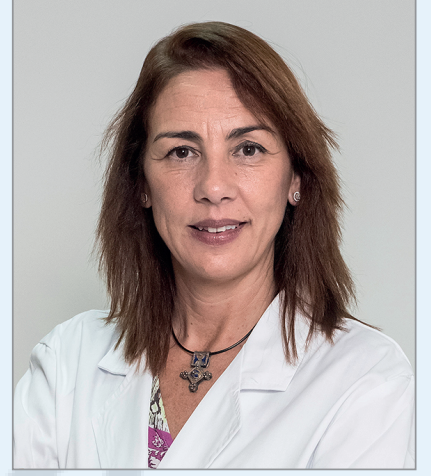

Lola Murillo

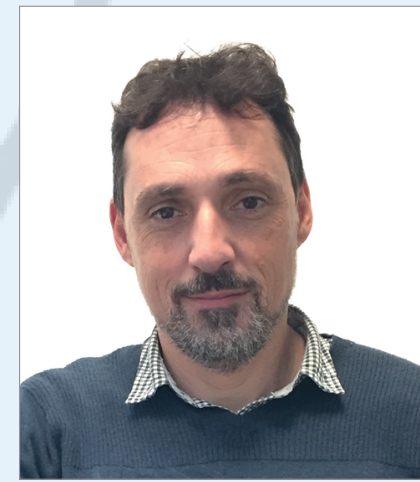

José Miguel Ruiz

\section{PALABRAS CLAVE}

Cronicidad, atención primaria, adherencia, polimedicación, coordinación

\section{KEYWORDS}

Chronicity, primary healthcare, adherence, multiple medication, coordination

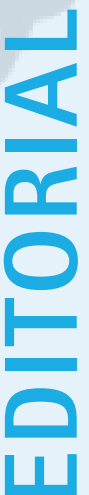

Las enfermedades crónicas son el gran reto de los sistemas sanitarios en la actualidad. Esta afirmación parece un mantra de los últimos tiempos que, a su vez, se está convirtiendo también en una premisa igualmente crónica, pues son muchos los años que se lleva hablando del problema que puede suponer el aumento de estas enfermedades para la sostenibilidad de la sanidad tal y como la entendemos hoy en día en los países desarrollados. No cabe duda, los datos demuestran que este reto ya está aquí. Las enfermedades crónicas representan el patrón epidemiológico dominante, pues en la actualidad suponen el 80 por ciento de las consultas en atención primaria y el 60 por ciento de las estancias hospitalarias en España, además del 80 por ciento del gasto sanitario. En total, más de 20 millones de personas tienen algún tipo de patología crónicas en nuestro país y a esto hay que añadir que el 50 por ciento de estas personas no son adherentes a su tratamiento, con el coste que esto puede tener en términos de salud y de eficiencia sobre el uso de los recursos disponibles.

Pese a todo esto y la reiterativa apelación a la importancia de abordar la cronicidad y poner a los pacientes en el centro del sistema, el modelo de atención sanitaria actual es principalmente reactivo y sigue estructurado alrededor de un patrón epidemiológico centrado mayoritariamente en las intervenciones agudas. Esto no se corresponde con las necesidades de los sistemas nacionales de salud actuales, que están obligados a atender, cada vez más, a personas con enfermedades crónicas, con una alta esperanza de vida y que quieren ser principalmente atendidas cerca de sus domicilios, cuando no en los mismos (especialmente si estas personas tienen algún tipo de dependencia o falta de movilidad). De hecho, el Observatorio Europeo de Sistemas y Políticas de Salud señala que "los proveedores de salud aún no han sido capaces de adaptar los programas de prevención y los programas de manejo de enfermedad a la realidad que hoy suponen las enfermedades crónicas". Adicionalmente, falta una integración del sistema sanitario con los recursos sociales asociados a la salud, que tienen una importancia sustancial para los enfermos crónicos.

Por si esto fuera poco, los pacientes tienen todavía un protagonismo episódico. Sin embargo, si se quiere tener éxito en cualquier acción enfocada a mejorar el abordaje de la cronicidad es necesaria la implicación de los pacientes en el cuidado de su propia salud. Desde hace décadas el sistema se ha construido con una lógica de salvar vidas y por ello está centrado en las enfermedades agudas. Ante el avance de las enfermedades crónicas es necesario complementar ese sistema con uno que se mueva tanto en términos de cuidar como de curar y ofrezca continuidad de cuidados durante toda una vida, con el potencial añadido de prevenir hospitalizaciones innecesarias y reducir costes. Se trata de optimizar recursos llevando a cabo intervenciones en las que el balance coste-efectividad sea positivo, por lo que es básico actuar sobre una población diana correctamente seleccionada. Las personas sanas o con factores de riesgo conocidos para la adquisición y desarrollo de enfermedades deberán ser orientadas a la responsabilidad en su propia salud y recibir formación en actividades preventivas. Aquellos pacientes con una enfermedad crónica deben recibir apoyo para favorecer su autogestión en los casos de las enfermedades menos complejas. En los pacientes de mayor complejidad y/o pluripatología es necesaria una gestión integral del caso en su conjunto, con cuidados fundamentalmente profesionales, dirigidos a los pacientes así como a su entorno y cuidadores.

En todo caso, los pacientes con patologías crónicas conviven con su enfermedad desde el día que se les detecta (incluso antes sin saberlo) hasta su fallecimiento por estas patologías $u$ otras causas. La enfermedad se convierte, por tanto, no en algo que interrumpe bruscamente la existencia normal de las personas (enfermedad aguda) sino en un contratiempo con el que hay que aprender a 
vivir. Por tanto, los cuidados sanitarios han de ofrecerse en el contexto en que el paciente desarrolla su vida habitual, es decir, en su propio entorno. Esto significa que el eje de dicha atención debería estar en la atención primaria y comunitaria y no en el hospital (en la atención primaria el 40 por ciento de los pacientes pluripatológicos tiene 3 o más enfermedades y el 94 por ciento está polimedicado). Además el rol del sistema sanitario cambia, ya no "elimina las competencias" sobre la vida del paciente, como ocurre en los hospitales, sino que se convierte en un acompañante que ayuda al paciente a tomar las decisiones adecuadas y vigila su salud para detectar cuando empieza a desviarse de los objetivos de salud, en cuyo caso es necesario actuar para evitar que haya consecuencias clínicas negativas. En el entorno del paciente hay bastantes agentes, muchos de ellos no sanitarios, que influyen en su salud: familiares, trabajo, asociaciones vecinales, residencias... Y todos esos activos en salud han de estar coordinados para ejercer la mejor influencia posible. En este contexto es más necesario que nunca crear puentes que puedan facilitar esa coordinación entre todos los agentes implicados, en mayor o menor medida, en la atención de la población con enfermedades crónicas.

Por tanto, si se quiere superar el reto que supone la cronicidad y mantener una atención de calidad a la población ahora y, especialmente, en los próximos años (en los que España será uno de los países más envejecidos del mundo, lo que previsiblemente traerá consigo más prevalencia de enfermedades crónicas y polimedicación) el sistema sanitario no puede ofrecer solamente un seguimiento y unos cuidados discontinuos a los pacientes crónicos en función de las agudizaciones o descompensaciones de sus patologías. Un buen abordaje de la cronicidad y de la adherencia terapéutica requiere fomentar el trabajo en equipos interdisciplinares, formados por los diferentes profesionales de los servicios sanitarios (entre ellos los farmacéuticos comunitarios, evidentemente). Los gestores y administradores del sistema tienen que asegurar la utilización más eficiente y equitativa de los recursos públicos puestos a su disposición y los profesionales deben participar activamente en lograr la disminución de la variabilidad de la práctica clínica y en la búsqueda del equilibro entre los objetivos de calidad y eficiencia del sistema. Independientemente de la adaptación de elementos de los diferentes modelos de provisión de cuidados crónicos, existen intervenciones organizativas prioritarias en la mejora del abordaje de la cronicidad entre las que destaca el fortalecimiento de los equipos de atención primaria y la reorganización de la atención.

\section{Modelo de coordinación}

Precisamente, para avanzar en una atención de la cronicidad más coordinada en el ámbito de la atención primaria y comunitaria y edificar esos puentes de colaboración, la Sociedad Española de Farmacia Familiar y Comunitaria (SEFAC), la Sociedad Española de Médicos de Atención Primaria (SEMERGEN), la Sociedad Española de Medicina de Familia y Comunitaria (semFYC) y la Sociedad Española de Médicos Generales y de Familia (SEMG) han publicado recientemente el documento Modelo de coordinación médico-farmacéutico para la atención al paciente con enfermedad crónica en atención primaria. Es la primera vez que expertos de estas sociedades trabajan conjuntamente en un documento de estas características, que pretende ser un punto de partida para mejorar el abordaje colaborativo del reto de la cronicidad desde un punto de vista realista y práctico. Hasta la fecha es cierto que han existido experiencias de colaboración entre médicos y farmacéuticos, pero más de forma aislada y esporádica que de una forma integrada y respaldada institucionalmente. Este documento persigue dar pautas para extender y generalizar lo que hasta ahora forma parte más de la voluntad individual.

Para alcanzar sus objetivos, el modelo de coordinación planteado en este documento busca cumplir los siguientes requisitos:

- Ser compatible con el trabajo diario de los profesionales.

- Ser atemporal.

- Ser aplicable independientemente de la patología que sufra el paciente.

- Ser capaz de promover la comunicación y la confianza entre profesionales.

- Ser capaz de medir resultados en salud y económicos.

- Ser capaz de integrar a enfermería y a otros profesionales sin los que no puede concebirse la atención adecuada al paciente crónico, y contar también con el respaldo de las asociaciones de pacientes.

El documento analiza las debilidades, amenazas, fortalezas y oportunidades que en la actualidad se dan en el sistema sanitario español en la línea de las ideas expuestas anteriormente en este texto. Además, se estudian los aspectos clínicos de la colaboración entre médicos y farmacéuticos, incidiendo en la existencia de dos niveles de actuación (pilotajes o experiencias locales y experiencias apoyadas por la Administración) y dos tipos de intervenciones, que pueden ser bien sobre el tratamiento (interacciones y efectos adversos, duplicidades, desprescripción, etc.) e intervenciones preventivas y de promoción de la salud como, por ejemplo, los cribados. También se recogen los requisitos necesarios para poner en práctica este modelo colaborativo. Estos requisitos tienen que ver con aumentar la confianza entre médico y farmacéutico y con la compatibilidad con la rutina de cada uno de estos profesionales, incidiendo en la gestión del tiempo, la comunicación y el uso de herramientas como los protocolos de trabajo consensuados. Dichos protocolos deben recoger información relacionada con su justificación, objetivos, población diana, sistema de registro, cronograma para su puesta en marcha, recursos necesarios, etc. y se actualizarían periódicamente.

El propósito de las sociedades firmantes del documento es que este éste se pueda llevar a la práctica y cuente, no solo con el respaldo de otros profesionales necesarios y de los pacientes, sino de las administraciones sanitarias. Es necesario dar un paso adelante y hacer lo que parece que en la teoría todos sabemos desde hace años: que la cronicidad ha llegado para quedarse y que no hay tiempo que perder si queremos que el sistema sanitario siga siendo de calidad, eficiente, equitativo y sostenible. Desde SEFAC, SEMERGEN, semFYC y SEMG esperamos que este documento sirva de ejemplo de esos puentes necesarios que entre todos debemos edificar para mejorar la comunicación con las personas, personalizar el trato y humanizar los cuidados; tres aspectos que deberían ser el hilo conductor en todas las intervenciones destinadas a mejorar el abordaje de la cronicidad de ahora en adelante. 